\title{
Kaon Pair Production in Proton-Proton Collisions
}

\author{
Y. Maeda,, , 困 M. Hartmann, ${ }^{2, \text {, }}$ I. Keshelashvili, ${ }^{2,3}$ S. Barsov, ${ }^{4}$ M. Büscher, ${ }^{2}$ M. Drochner, ${ }^{5}$ \\ A. Dzyuba, ${ }^{2,4}$ V. Hejny, ${ }^{2}$ A. Kacharava, ${ }^{3,6}$ V. Kleber, ${ }^{7}$ H.R. Koch, ${ }^{2}$ V. Koptev, ${ }^{4}$ P. Kulessa, ${ }^{8}$ \\ B. Lorentz, ${ }^{2}$ T. Mersmann, ${ }^{9}$ S. Mikirtytchiants, ${ }^{2,4}$ A. Mussgiller,${ }^{6}$ M. Nekipelov, ${ }^{2,}{ }^{4}$ H. Ohm, ${ }^{2}$ \\ D. Prasuhn, ${ }^{2}$ R. Schleichert,${ }^{2}$ H.J. Stein,${ }^{2}$ H. Ströher,${ }^{2}$ Yu. Valdau, ${ }^{2,4}$ C. Wilkin, ${ }^{10}$ and P. Wüstner ${ }^{5}$ \\ ${ }^{1}$ Research Center for Nuclear Physics, Osaka University, Ibaraki, Osaka 567-0047, Japan \\ ${ }^{2}$ Institut für Kernphysik, Forschungszentrum Jülich, 52425 Jülich, Germany \\ ${ }^{3}$ High Energy Physics Institute, Tbilisi State University, 0186 Tbilisi, Georgia \\ ${ }^{4}$ High Energy Physics Department, Petersburg Nuclear Physics Institute, 188350 Gatchina, Russia \\ ${ }^{5}$ Zentralinstitut für Elektronik, Forschungszentrum Jülich, 52425 Jülich, Germany \\ ${ }^{6}$ Physikalisches Institut II, Universität Erlangen-Nürnberg, 91058 Erlangen, Germany \\ ${ }^{7}$ Physikalisches Institut, Universität Bonn, 53115 Bonn, Germany \\ ${ }^{8}$ H. Niewodniczański Institute of Nuclear Physics PAN, 31342 Kraków, Poland \\ ${ }^{9}$ Institut für Kernphysik, Universität Münster, 48149 Münster, Germany \\ ${ }^{10}$ Physics and Astronomy Department, UCL, Gower Street, London WC1E 6BT, UK
}

(Dated: February 11, 2013)

\begin{abstract}
The differential and total cross sections for kaon pair production in the $p p \rightarrow p p K^{+} K^{-}$reaction have been measured at three beam energies of $2.65,2.70$, and $2.83 \mathrm{GeV}$ using the ANKE magnetic spectrometer at the COSY-Jülich accelerator. These near-threshold data are separated into pairs arising from the decay of the $\phi$-meson and the remainder. For the non- $\phi$ selection, the ratio of the differential cross sections in terms of the $K^{-} p$ and $K^{+} p$ invariant masses is strongly peaked towards low masses. This effect can be described quantitatively by using a simple ansatz for the $K^{-} p$ final state interaction, where it is seen that the data are sensitive to the magnitude of an effective $K^{-} p$ scattering length. When allowance is made for a small number of $\phi$ events where the $K^{-}$rescatters from the proton, the $\phi$ region is equally well described at all three energies. A very similar phenomenon is discovered in the ratio of the cross sections as functions of the $K^{-} p p$ and $K^{+} p p$ invariant masses and the identical final state interaction model is also very successful here. The world data on the energy dependence of the non- $\phi$ total cross section is also reproduced, except possibly for the results closest to threshold.
\end{abstract}

PACS numbers: 13.60.Le, 14.40.Aq, 25.40.Ep

\section{INTRODUCTION}

There are several mechanisms that can lead to the production of kaon-antikaon pairs in nucleon-nucleon collisions near threshold. These can be divided mainly into two general classes involving (a) the production of a nonstrange meson that subsequently decays into $K \bar{K}$, and (b) the associated production of $K Y^{*}$, where the $\bar{K}$ is formed through the decay of the hyperon $Y^{*}$. The most prominent meson that is found almost exclusively in the $K^{+} K^{-} / K^{0} \bar{K}^{0}$ channels is, of course the $\phi$, and there have been measurements of the $p p \rightarrow p p \phi \rightarrow p p K^{+} K^{-}$ reaction from DISTO [1] and COSY-ANKE [2], as well as of the $p n \rightarrow d \phi \rightarrow d K^{+} K^{-}$reaction [3]. These experiments show strength also away from the region of the $\phi$ peak that could arise from other mesons decaying into $K^{+} K^{-}$.

The lightest of the non-strange scalar mesons are the $a_{0}(I=1)$ and $f_{0}(I=0)$, which decay mainly into $\eta \pi$ and $\pi \pi$, respectively. Both have masses around $980 \mathrm{MeV} / \mathrm{c}^{2}$ and widths of the order of $50-100 \mathrm{MeV} / \mathrm{c}^{2}$ [4]. Since

\footnotetext{
*E-mail: ymaeda@rcnp.osaka-u.ac.jp

†E-mail: M.Hartmann@fz-juelich.de
}

the central mass values fall almost exactly at the $K \bar{K}$ threshold, the strong coupling to this channel distorts significantly the upper parts of the mass spectra [5]. It has even been suggested that both resonances might be mainly molecular in nature [6]. Since the $K^{+} K^{-}$system is a mixture of isospin $I=0$ and $I=1$, one can consider whether the non- $\phi$ events observed in $p p \rightarrow p p K^{+} K^{-}$ might be linked to the production and decay of the $a_{0}$ and/or the $f_{0}$ mesons. The $I=1$ channel is isolated cleanly by looking at the $p p \rightarrow d K^{+} \bar{K}^{0}$ reaction [7].

On the other hand, we can also expect kaon pairs to be created in the $p p \rightarrow K^{+} p Y^{*}$ reaction, where the hyperon decays through $Y^{*} \rightarrow K^{-} p$. There are, of course, several excited hyperons that could contribute to such a process. Of particular interest for low energy production are the $\Sigma(1385)$ and the $\Lambda(1405)$. Though nominally lying below the sum of the $K^{-}$and $p$ masses, their large widths $\left(\sim 50 \mathrm{MeV} / \mathrm{c}^{2}\right)$ ensure that they overlap the $K^{-} p$ threshold [4]. Results have recently been presented on the production of these states in the $p p \rightarrow$ $K^{+} p \Sigma^{0}(1385)$ and $p p \rightarrow K^{+} p \Lambda(1405)$ reactions, where the hyperons were detected in the $\Lambda \pi^{0}$ and $\Sigma^{0} \pi^{0}$ channels, respectively [8].

In addition to being involved in a direct production mechanism, the $\Lambda(1405)$ and the $\Sigma(1385)$ might also lead to a significant $K^{-} p$ final state interaction $(f s i)$ in the 
$p p \rightarrow p p K^{+} K^{-}$reaction. Evidence that the $K^{-} p \quad f s i$ is indeed important is to be found in the measurement of the $p p \rightarrow p p K^{+} K^{-}$cross section at energies below the $\phi$ threshold [9]. It was shown that the invariant masses of the $K^{-} p$ final system were on average lower than those of the $K^{+} p$ pairs and it was suggested that this is due to the strong attraction in the $K^{-} p$ system.

It is therefore an interesting but open question whether the $p p \rightarrow p p K^{+} K^{-}$data from the non- $\phi$ region should be viewed, in first approximation, as scalar resonance production via $p p \rightarrow p p a_{0} / f_{0}$ or hyperon production through $p p \rightarrow K^{+} p \Lambda(1405) / \Sigma^{0}(1385)$. It is one aim of the present paper to study this competition by using the data obtained when investigating $\phi$-meson production in proton-proton collisions at ANKE [2].

A program to investigate the production of kaon pairs in nucleon-nucleon collisions has been initiated at the COSY storage ring of the Forschungszentrum Jülich. Although the methods of the measurement and analysis of the $p p \rightarrow p p K^{+} K^{-}$reaction have already been given in Ref. 2], for the sake of clarity the main points are repeated in Sect I. Due to the finite width of the $\phi$, distinguishing between events corresponding to the production of this meson and the rest requires a detailed modelling of the $K^{+} K^{-}$spectrum, as described in Sect. III. When more than one pair of particles interact strongly in the final state, there is no reliable prescription to evaluate a corresponding enhancement factor. Our approach to this problem in terms of an effective $K^{-} p$ scattering length $a$ is described in Sect. [V] with a heuristic justification being given in Appendix $\mathrm{A}$

The relative distributions of the $K^{-} p$ and $K^{+} p$ invariant masses are shown and discussed in Sect. V] The ratio of the differential cross sections as functions of the $K^{-} p$ and $K^{+} p$ invariant masses indicates that the $K^{-} p$ attraction is very strong. This confirms the earlier COSY-11 findings [9] but with higher statistics over much wider ranges of energy and mass. The events that come from the non- $\phi$ region in the $2.65 \mathrm{GeV}$ data allow us to fix the parameter $a$ necessary to describe the $K^{-} p / K^{+} p$ ratio within our final state interaction model. The data are mainly sensitive to the magnitude $|a|$ and the value obtained is not dissimilar to that required to describe free $K^{-} p$ scattering.

The approach reproduces also our results at the other two energies as well as the COSY-11 data. If allowance is made for the small possible contribution to this ratio from $\phi$ events, of the order of $20 \%$, the two-particle ratio is also well described for all $K^{+} K^{-}$mass intervals. In addition, we find a completely analogous effect in the three-body $K p p$ system, with the $K^{-} p p / K^{+} p p$ ratio being strongly biased towards the lowest values of the invariant mass. We show in Sect. VI that these distributions are also predicted quantitatively by the same $K^{-} p$ final state interaction model with the same value of the parameter $a$.

The total cross sections for $\phi$ and non- $\phi$ production are presented in Sect. VII The energy dependence of the non- $\phi$ total cross section is strongly influenced by both the $p p$ and $K^{-} p$ fsi but, even after including these effects, the lowest energy data are not well reproduced. This leads to the suggestion that the effect might be connected with the two or three points in the $K^{+} K^{-}$spectrum at very low invariant masses, which lie consistently above the simulations. Our conclusions and views on the outlook are presented in Sect. VIII.

\section{EXPERIMENT AND DATA ANALYSIS}

The study of kaon pair production has been performed using data taken with the ANKE magnetic spectrometer [10]. This is placed at an internal target station of COSY, which is the COoler SYnchrotron of the Research Center Jülich [11]. We have already reported an initial analysis of the $p p \rightarrow p p K^{+} K^{-}$data, from which values of the $p p \rightarrow p p \phi$ cross sections were extracted [2].

The measurements were performed at three proton kinetic energies of $T_{p}=2.65 \mathrm{GeV}, 2.70 \mathrm{GeV}$ and $2.83 \mathrm{GeV}$, corresponding to excess energies of $\varepsilon=51 \mathrm{MeV}, 67 \mathrm{MeV}$ and $108 \mathrm{MeV}$ with respect to the $p p K^{+} K^{-}$threshold. A dense hydrogen cluster jet target [12] provided areal densities of $\sim 5 \times 10^{14} \mathrm{~cm}^{-2}$ and the average luminosity during the experiment was typically $2.3 \times 10^{31} \mathrm{~cm}^{-2} \mathrm{~s}^{-1}$. The detection system of the three-dipole magnetic spectrometer ANKE registers simultaneously slow positively and negatively charged particles, with fast positive particles being measured in the forward system [10, 13, 14].

Kaons produced in the $p p \rightarrow p p K^{+} K^{-}$reaction at 2.65 and $2.70 \mathrm{GeV}$ have laboratory momenta between 0.2 and $1.0 \mathrm{GeV} / \mathrm{c}$ and this full range was covered by the detection system for both $K^{+}$and $K^{-}$. For the $2.83 \mathrm{GeV}$ run, the $K^{+}$were detected only up to $0.6 \mathrm{GeV} / \mathrm{c}$, which reduced the geometrical acceptance. The momentum range of the protons emerging from the reaction is between 0.8 and $1.6 \mathrm{GeV} / \mathrm{c}$, for which the forward detector has full acceptance.

The momenta of both the kaons and protons were determined from the track information furnished by sets of multiwire proportional chambers. The tracking efficiency for kaons fell smoothly from $98 \%$ to $93 \%$ as the momentum increased, whereas that for protons was about $80 \%$ over the whole momentum range. The efficiency map, including its dependence on the particle momenta and position, was generated for the track reconstruction and used in the analysis. The data-taking efficiency was around $90 \%$ and, taken in combination with the tracking efficiency, resulted in a total detector efficiency of about $60 \%$.

In order not to reduce the geometric acceptance unnecessarily, only one forward-going proton was measured in coincidence with the charged kaon pair. The $p p \rightarrow p p K^{+} K^{-}$reaction was then identified by requiring that the missing mass in the reaction was consistent with that of the non-observed proton. As a first step, positive kaons were selected through a procedure, described in 
detail in Ref. [15], that used the time of flight (TOF) between START and STOP scintillation counters of a dedicated $K^{+}$detection system. In the second stage, both the $K^{-}$and forward-going proton were identified from the time-of-flight differences between the STOP counters in the negative and forward detector systems with respect to the STOP counter in the positive system that was hit by the $K^{+}$. By comparing this with the time difference expected for a kaon pair based upon their measured momenta, a clear separation is achieved, with good events lying in an island where the two time differences are consistent [14]. These two TOF selections, as well as that for the $K^{+}$, were carried out within $\pm 3 \sigma$ bands.

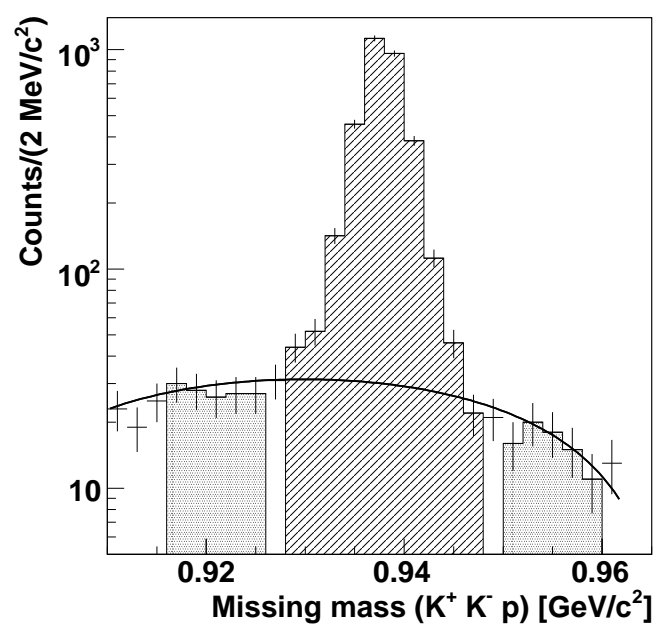

FIG. 1: Missing-mass distribution of $p p \rightarrow K^{+} K^{-} p X$ events at $T_{p}=2.65 \mathrm{GeV}$. The hatching indicates the range used for the selection of protons. The side-band events scaled by the solid line are indicated by the dotted shading.

The spectrum of missing masses with respect to the $K^{+} K^{-} p$ system, shown in Fig. 1 1 for the $2.65 \mathrm{GeV}$ data, is dominated by the proton peak. The corrected numbers of $K^{+} K^{-} p p$ events obtained in this way are around 3100 (at $\left.T_{p}=2.65 \mathrm{GeV}\right), 1300(2.70 \mathrm{GeV})$, and $650(2.83 \mathrm{GeV})$. The estimated background inside the proton cut window is $5 \%, 12 \%$, and $18 \%$ at these three energies, respectively. This has been subtracted using the side-band events scaled by the solid line shown in Fig. 1. The uncertainty resulting from this procedure is estimated to be less than $3 \%$ for the total cross sections and negligible compared to the statistical errors for the differential distributions.

After the identification of the $p p \rightarrow p p K^{+} K^{-}$events, the data were binned in intervals of the $K^{+} K^{-}$invariant mass, with the $2.65 \mathrm{GeV}$ results being presented in Fig. 2 . This spectrum shows a very prominent $\phi$ peak positioned close to the nominal mass of the meson. In addition there is a non- $\phi$ contribution that decreases steadily from low to high invariant masses. This behavior, which is mainly a reflection of the ANKE acceptance for non-resonant $p p K^{+} K^{-}$production, has to be simulated in order to derive cross sections.

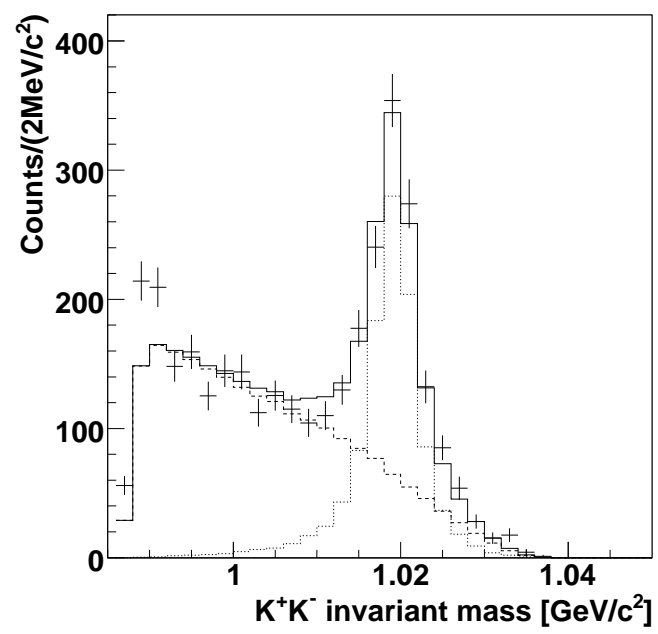

FIG. 2: The $K^{+} K^{-}$invariant-mass distribution for the $p p \rightarrow p p K^{+} K^{-}$reaction at $2.65 \mathrm{GeV}$. The prominent $\phi$ peak sits on a steadily falling background corresponding to the ANKE acceptance for the four-particle final state. The simulation of these two contributions and their sum are shown, respectively, by the dotted, dashed, and solid histograms.

The acceptance has been determined through a MonteCarlo simulation based upon GEANT4 [16], where the geometrical acceptance, resolution, detector efficiency, and kaon decay probability were taken into account. In the initial step, the $\phi$-production distributions previously published 2] were taken as the basis of the simulation, whereas four-body phase space was used for the non- $\phi$ component of Fig. 2. There are seven degrees of freedom for a four-body final state produced in an unpolarized reaction. Thus for each beam energy, seven independent c.m. distributions were generated (five angular distributions, the relative momentum of final protons, and the $K^{+} K^{-}$invariant mass). These were then divided into two groups depending on the value of the $K^{+} K^{-}$invariant mass, i.e., $\phi$-rich and $\phi$-poor regions. All distributions were jointly fitted to the experimental data and the relative contribution of $\phi$ and non- $\phi$ production evaluated for the determination of the acceptances.

In the $\phi$-poor region, most of the c.m. distributions are fairly uniform, though the polar angle of the $K^{+} K^{-}$ system relative to the beam axis in the overall c.m. system shows deviations. Fitting this with the function $1+\alpha \cos ^{2} \theta$ gives anisotropies of $\alpha=0.5 \pm 0.2$ and $\alpha=1.5 \pm 0.5$ at $2.65 \mathrm{GeV}$ and the two higher energies, respectively. The inclusion of this factor increases the total acceptance for non- $\phi$ production by about $10 \%$. In addition, as will be seen in Sects. V] and VI the $K^{ \pm} p$ and $K^{ \pm} p p$ invariant masses deviate strongly from phase space. These deviations have been taken into account in the simulations, using iteratively the theoretical approach discussed in Sect. IV] in order to converge on the acceptance-corrected distributions. The simulations of 
the $\phi$ and non- $\phi$ components of the uncorrected $K^{+} K^{-}$ spectrum at $2.65 \mathrm{GeV}$ are shown in Fig. 2 together with their sum.

The estimated total acceptance for kaon-pair production is $1.1 \%(2.65 \mathrm{GeV}), 0.7 \%(2.70 \mathrm{GeV})$ and $0.1 \%$ $(2.83 \mathrm{GeV})$, and the associated systematic uncertainties in the total cross section are $5 \%, 7 \%$ and $12 \%$, respectively. The shapes of the differential cross sections, especially those for the $K p$ and $K p p$ invariant masses, are very stable to this iterative procedure. As a consequence, the uncertainty that this introduces in the differential cross sections is negligible compared to the statistical errors.

The luminosity required to evaluate absolute cross sections was determined by measuring in parallel protonproton elastic scattering for laboratory angles between $5.0^{\circ}$ and $8.5^{\circ}$. For this purpose the momentum of a forward-going proton was determined using the ANKE forward detector. After a missing-mass analysis, the (small) background underneath the proton peak was subtracted and the remaining events were corrected for efficiency and acceptance.

\section{THE $K^{+} K^{-}$INVARIANT MASS DISTRIBUTION}

Figure 3 shows the $p p \rightarrow p p K^{+} K^{-}$differential cross sections in terms of the $K^{+} K^{-}$invariant masses as obtained at our three energies. These have been corrected for acceptance, deadtime, etc., and normalized on the basis of the proton-proton elastic scattering data, as discussed in Sect. VII] The resulting non- $\phi$ contribution is much flatter than that illustrated in Fig. 2 In order to ensure a clean separation of the $\phi$ from the rest, it is necessary to model the shapes as reliably as possible.

In our earlier work [2], the non- $\phi$ contribution was assumed to be described by a four-body phase space. The $\phi$ was taken to have a Breit-Wigner form with a constant width of $\Gamma=4.26 \mathrm{MeV} / \mathrm{c}^{2}$ [4], which was then convoluted with an experimental resolution of $\sigma=1 \mathrm{MeV} / \mathrm{c}^{2}$. Only for the $\phi$ component was the spectrum modified to include effects arising from the final state interaction between the emerging protons. These three assumptions have now to be reconsidered.

Since the $\phi$ is a $p$-wave resonance that decays strongly into $K \bar{K}$, its width depends upon the $K^{+} K^{-}$and $K^{0} \bar{K}^{0}$ relative momenta. Due to the narrowness of the state, the peak itself is little affected by this refinement but it does suppress slightly the low mass tail of the resonance. Although for low $K^{+} K^{-}$effective masses there is no clear evidence for any $p p$ fsi, only minor effects are to be expected there because of the large amount of energy that is then available to excite the $p p$ system. Nevertheless, the $f$ si cannot be neglected at higher $K^{+} K^{-}$masses, where its inclusion distorts slightly the four-body phase space spectrum. Finally, as will be seen in Sect. [V] it is clear that there is a large final state interaction between

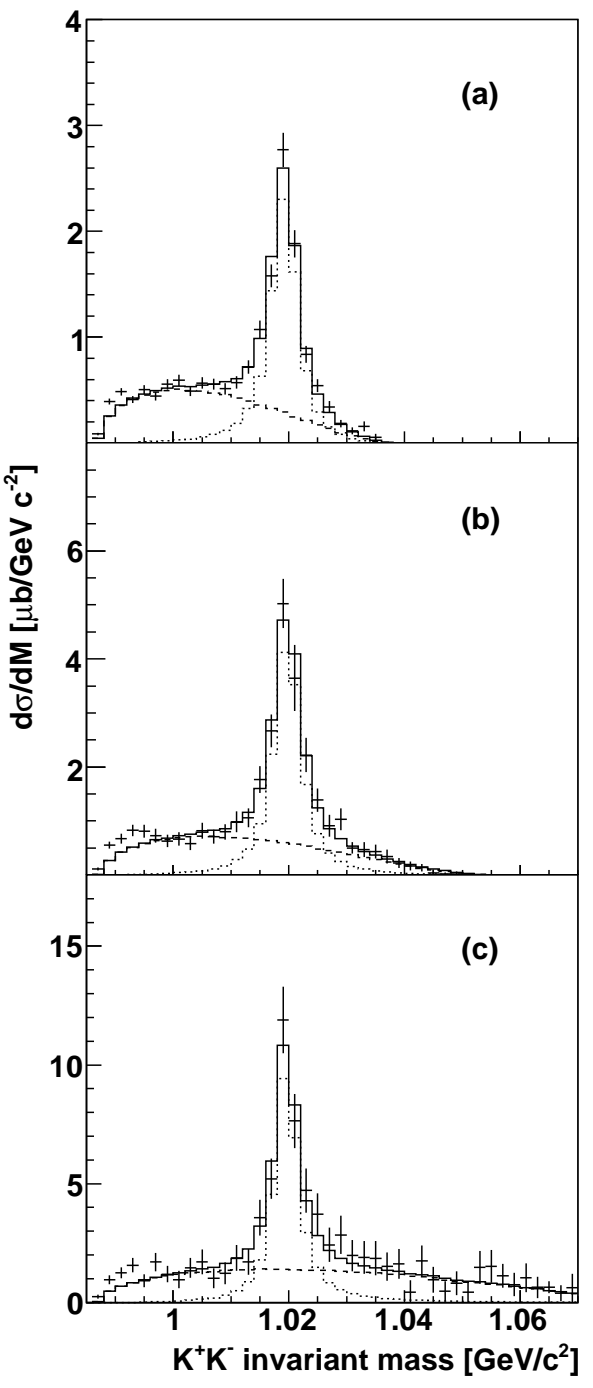

FIG. 3: Differential cross section for the $p p \rightarrow p p K^{+} K^{-}$ reaction with respect to the $K^{+} K^{-}$invariant mass at (a) $2.65 \mathrm{GeV}$, (b) $2.70 \mathrm{GeV}$, and (c) $2.83 \mathrm{GeV}$. The dotted histogram and the dashed curve represent the simulations for the $\phi$ and non- $\phi$ contributions to the spectrum, with their sum being shown by the solid histogram. For all three energies the simulations fail to reproduce the lowest mass points.

the $K^{-}$and one or both protons and this also modifies the shape of the non- $\phi$ component, enriching the central part of the $K^{+} K^{-}$spectrum. By chance, all three effects go in the same direction. In the fits to Fig. 3, the fractions to be associated with $\phi$ production are diminished somewhat compared to those reported in our previous work [2].

It must be noted that the lowest mass points lie above the fitted curves for all three data sets of Fig. 3, These deviations from the simulations are significant because, as seen from the comparison of Figs. 2 and 3 a, the ANKE acceptance is largest in this region. Although ignored in 
the fitting process, they were taken into account in the evaluation of the non- $\phi$ contribution to the total cross section, contributing $4 \%(2.65 \mathrm{GeV}), 6 \%(2.70 \mathrm{GeV})$, and $10 \%(2.83 \mathrm{GeV})$.

\section{FINAL STATE INTERACTION ASSUMPTIONS}

We wish to describe the $p p \rightarrow p p K^{+} K^{-}$data at all energies in terms of final state interactions between the two protons and between the $K^{-}$and either one or both of the protons. There is no reliable way of carrying out such a program without solving multibody equations, which is well outside the scope of the present paper. As explained in Appendix A we make the ad hoc assumption that the overall enhancement factor is the product of the enhancements in the $p p$ and two $K^{-} p$ systems, all evaluated at the appropriate relative momenta $q$ :

$$
F=F_{p p}\left(q_{p p}\right) \times F_{K p}\left(q_{K p_{1}}\right) \times F_{K p}\left(q_{K p_{2}}\right) .
$$

Similar approaches are to be found elsewhere in the literature as, for example, in the description of the $p p \eta$ final state at low energies [17].

The $K^{-} p$ enhancement factor is taken in the scattering length approximation

$$
F_{K p}(q)=\frac{1}{1-i q a}
$$

where some caution should be exercised in the interpretation of the complex parameter $a$ as an effective scattering length. In principle $a$ could have an energy dependence, which would correspond to the introduction of an effective range term. However, it is hard from our data to determine the values of any extra free parameters.

A proton-proton enhancement factor of the form

$$
\left|F_{p p}(q)\right|^{2}=\frac{q^{2}+\beta^{2}}{q^{2}+\alpha^{2}}
$$

is assumed [18], where the position of the ${ }^{1} S_{0}$ virtual state is well fixed at $\alpha=0.1 \mathrm{fm}^{-1}$. The final state interaction should not influence higher partial waves and, in order to reduce its effects above about $10 \mathrm{MeV}$, we take $\beta=$ $0.5 \mathrm{fm}^{-1}$. The relative momentum of the two protons in the $p p$ rest frame, which is very sensitive to the form of the $p p f s i$, is then reproduced reasonably, as seen from Fig. 4 .

It is important to note that the form of neither the $p p$ nor the $K^{-} p$ enhancement factor is valid for an excitation energy below about $1 \mathrm{MeV}$ due to the explicit Coulomb force. However, these regions represent only very small fractions of the allowed phase space.

\section{THE KAON-PROTON INVARIANT-MASS DISTRIBUTION}

In data taken with the COSY-11 spectrometer at $\varepsilon=28 \mathrm{MeV}$, i.e. at an energy where there is very lit-

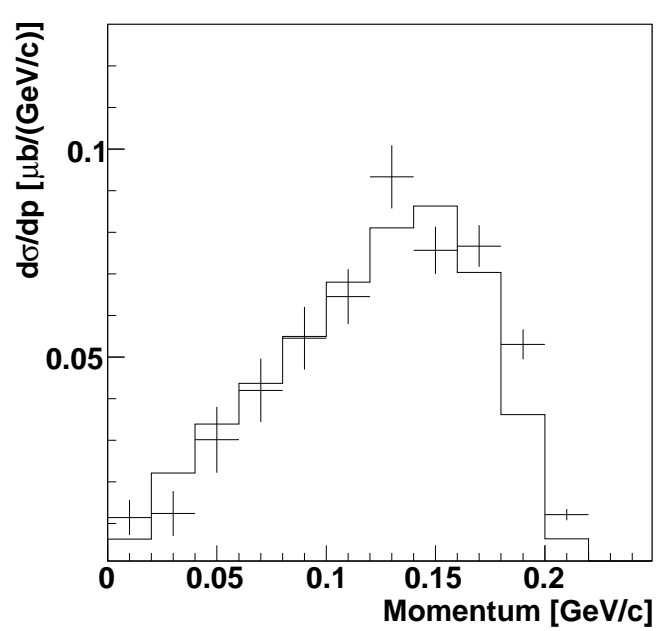

FIG. 4: Relative momentum of the final protons from the $p p \rightarrow p p K^{+} K^{-}$reaction in the $p p$ rest frame. The experimental points at $2.65 \mathrm{GeV}$ are compared to the simulation, using the standard parameters, which is shown as a histogram.

tle $\phi$ production, it was found that the $K^{-} p$ and $K^{+} p$ invariant-mass $M(K p)$ distributions were markedly different [9]. The ratio of the acceptance-corrected distributions

$$
R_{K p}=\frac{d \sigma / d M\left(K^{-} p\right)}{d \sigma / d M\left(K^{+} p\right)}
$$

showed a very strong preference for low values of $M(K p)$.

Figure 5 shows the $K p$ spectra obtained at our lowest energy. In order to consider a situation similar to that of COSY-11, the data have been selected such that only events with $K^{+} K^{-}$invariant masses less than $1.01 \mathrm{GeV} / \mathrm{c}^{2}$ are retained. The simulation that takes into account only the $p p f s i$ fails to describe the data. Furthermore, the ratio of the $K^{-} p$ to $K^{+} p$ mass distributions is far from constant, as it would be if there were only the $p p$ $f s i$. The peaking of $R_{K p}$ to the lowest invariant masses confirms the COSY-11 observation [9] but with higher statistics and over a wider range of $K p$ masses.

When the $K^{-} p$ final-state-interaction factors are also introduced into the four-body phase space simulation of the non- $\phi$ contribution to the $p p \rightarrow p p K^{+} K^{-}$reaction, the individual $d \sigma / d M\left(K^{+} p\right)$ and $d \sigma / d M\left(K^{-} p\right)$ distributions of Fig. [5 as well as their ratio $R_{K p}$, are all well described. The best fit to these data is achieved with an effective $K^{-} p$ scattering length of $a=(0+1.5 i) \mathrm{fm}$ and this value of $a$ will be retained for all the subsequent simulations. Nevertheless, the uncertainties in the real and imaginary parts are quite large and strongly correlated such that $|a|$ is determined much better than its phase, as illustrated by the $\chi^{2}$ contour plot of Fig. 6, The free $K^{-} p I=0$ and $I=1$ values from Ref. [19] are also shown there along with their one $-\sigma$ limits. From this it is seen that there is an overlap with either isospin scattering length. One has also to bear in mind that our 


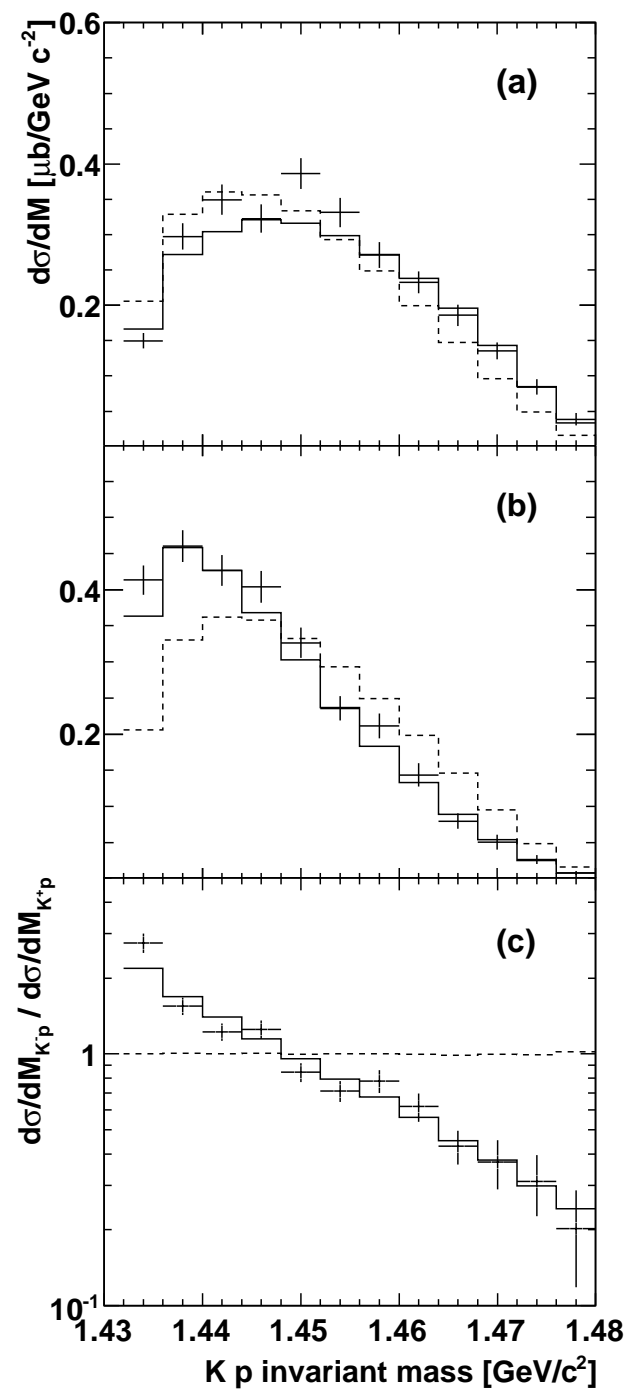

FIG. 5: Differential cross sections for the $p p \rightarrow p p K^{+} K^{-}$reaction with respect to the invariant mass of (a) the $K^{+} p$ and (b) the $K^{-} p$ systems. In panel (c) their ratio is shown on a logarithmic scale. These $2.65 \mathrm{GeV}$ data have been selected to come from the region $M\left(K^{+} K^{-}\right)<1.01 \mathrm{GeV} / \mathrm{c}^{2}$, where there is only a very small $\phi$ contribution. The dashed histograms represent the results of four-body phase-space simulations, where the only distortion is that coming from the $p p f s i$. The solid histograms include also the $K^{-} p$ fsi.

data represent averages over some energy range and that a constant scattering length is necessarily an oversimplification.

The situation for higher $K^{+} K^{-}$masses is complicated by the contribution from the $\phi$ meson. At our beam energies, the average separation of the $K^{-}$(produced in the $\phi$ decay) from one of the final protons is about $7 \mathrm{fm}$. This large distance reduces considerably the effects of the $K^{-} p f_{\text {si }}$. We would therefore expect that deviations of $R_{K p}$ from unity should arise primarily from the non- $\phi$

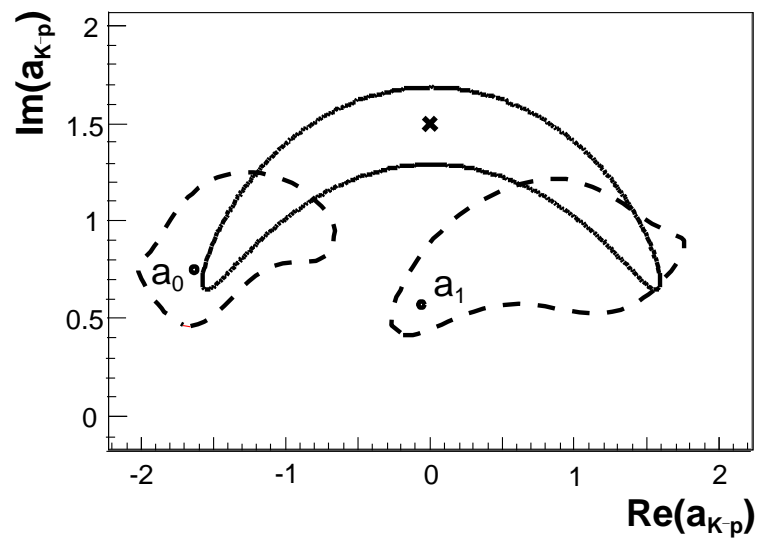

FIG. 6: Contour plot corresponding to the one- $\sigma$ level uncertainty of the fits of the complex parameter $a$ of the $f_{s i}$ ansatz to the data of Fig. [5 Our best fit is obtained with $a=1.5 i \mathrm{fm}$ (marked with a cross). Also shown are the best fit values (points) and corresponding contours (dashed lines) for the isoscalar $\left(a_{0}\right)$ and isovector $\left(a_{1}\right) K^{-} p$ scattering lengths derived from the study of free $K^{-}$-nucleon scattering [19].

contributions. The modelling of the data thus depends in the first instance upon the determination of the fraction of $\phi$ production in the overall $p p \rightarrow p p K^{+} K^{-}$spectra presented in Fig. 3. However, classical simulations suggest that more than $15 \%$ of the $\phi$ decays lead to at least one $K^{-} p$ pair with a separation of less than $1 \mathrm{fm}$, for which the $f_{s i}$ is not negligible.

To investigate this point further, the $2.65 \mathrm{GeV}$ data have been divided into four regions of $K^{+} K^{-}$invariant mass, viz. $M\left(K^{+} K^{-}\right)<0.995 \mathrm{GeV} / \mathrm{c}^{2}, 0.995<$ $M\left(K^{+} K^{-}\right)<1.003 \mathrm{GeV} / \mathrm{c}^{2}, 1.003<M\left(K^{+} K^{-}\right)<$ $1.010 \mathrm{GeV} / \mathrm{c}^{2}$, and $1.010 \mathrm{GeV} / \mathrm{c}^{2}<M\left(K^{+} K^{-}\right)$. The first two intervals contain almost no $\phi$ events, whereas the last corresponds mainly to $\phi$ production. The corresponding $R_{K p}$ distributions are shown in Fig. 7, where they are compared to the same simulation as that shown in Fig. 5. The non- $\phi$ region is very well described but, for high $K^{+} K^{-}$invariant masses, the asymmetry seems to be diluted too much by kaons from the $\phi$ decay. The agreement is improved by assuming that $20 \%$ of the $\phi$ events are influenced by the $K^{-} p f s i$. The same effect could be achieved by artificially reducing the fraction of $\phi$ mesons in the peak of Fig. 3a by $10 \%$, but this would be hard to justify.

If the strong $K^{-} p$ final state interaction is responsible for the distortion in the $K^{-} p / K^{+} p$ ratio seen in Fig. 7 , similar effects should be seen for the other excess energies. For our $\varepsilon=66.6$ and $108 \mathrm{MeV}$ results shown in Fig. 8, the statistics are lower and we merely divide each data set into two, comprising $\phi$-rich and $\phi$-poor regions. Without any change in the value of the $K^{-} p$ effective scattering length, all the distributions are well described, especially if it is assumed that $20 \%$ of the $\phi$ events lead to $K^{-} p$ fsi effects.

Data on the $K^{-} p / K^{+} p$ ratio were not published by 


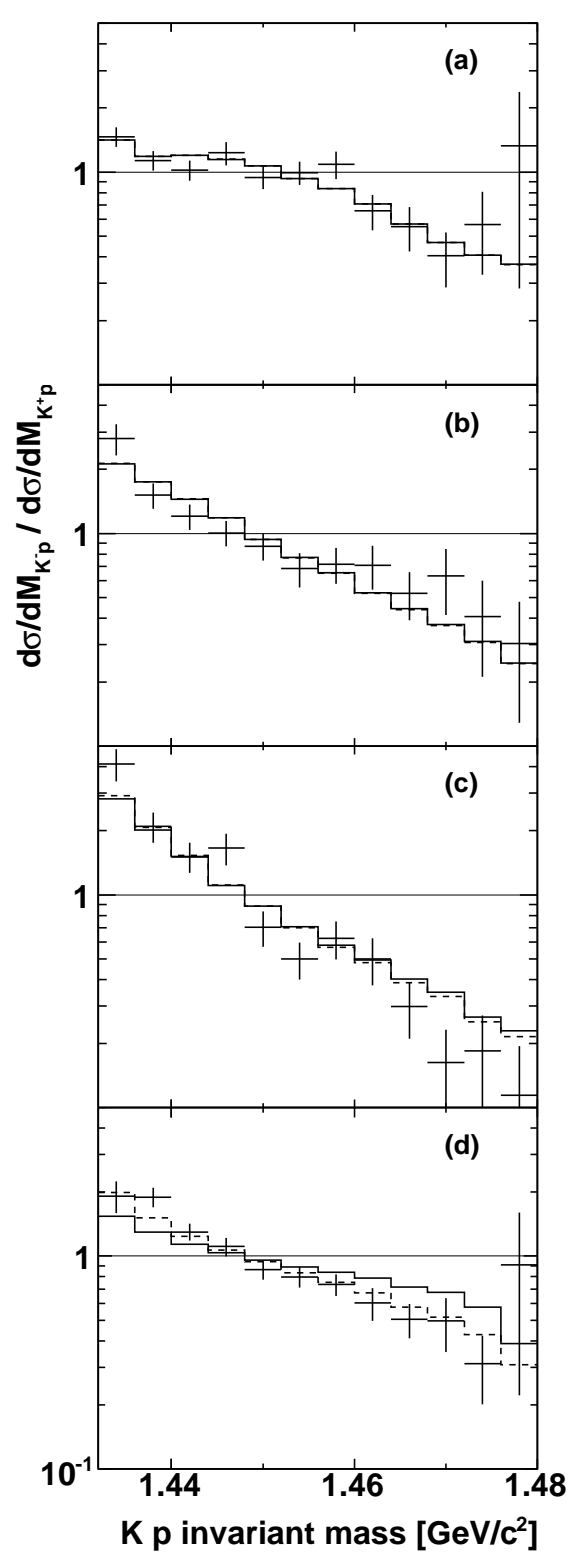

FIG. 7: Values of the $R_{K p}$ ratio for the $p p \rightarrow p p K^{+} K^{-}$ reaction at $2.65 \mathrm{GeV}$ extracted for four different selections of the $K^{+} K^{-}$invariant mass, viz. (a) $M\left(K^{+} K^{-}\right)<$ $0.995 \mathrm{GeV} / \mathrm{c}^{2}$, (b) $0.995<M\left(K^{+} K^{-}\right)<1.003 \mathrm{GeV} / \mathrm{c}^{2}$, (c) $1.003<M\left(K^{+} K^{-}\right)<1.010 \mathrm{GeV} / \mathrm{c}^{2}$, and (d) $1.010 \mathrm{GeV} / \mathrm{c}^{2}<$ $M\left(K^{+} K^{-}\right)$. The histograms are predictions of the model described in the text, where the $K^{-}$mesons from non- $\phi$ events are subject to a $K^{-} p$ final state interaction. In the high mass interval, the effects of assuming a $20 \%$ probability of the $\mathrm{K}^{-}$ from the $\phi$ decay being influenced by a $f s i$ is considered and this leads to the dashed histograms.

the DISTO collaboration [1]. However, the same good description of $R_{K p}$ is found for the COSY-11 results of Fig. 9 where, in view of the low excess energies, there is no ambiguity regarding the $\phi$ contribution. Given that the systematic uncertainties in this experiment are independent of those at ANKE, the quantitative agreement
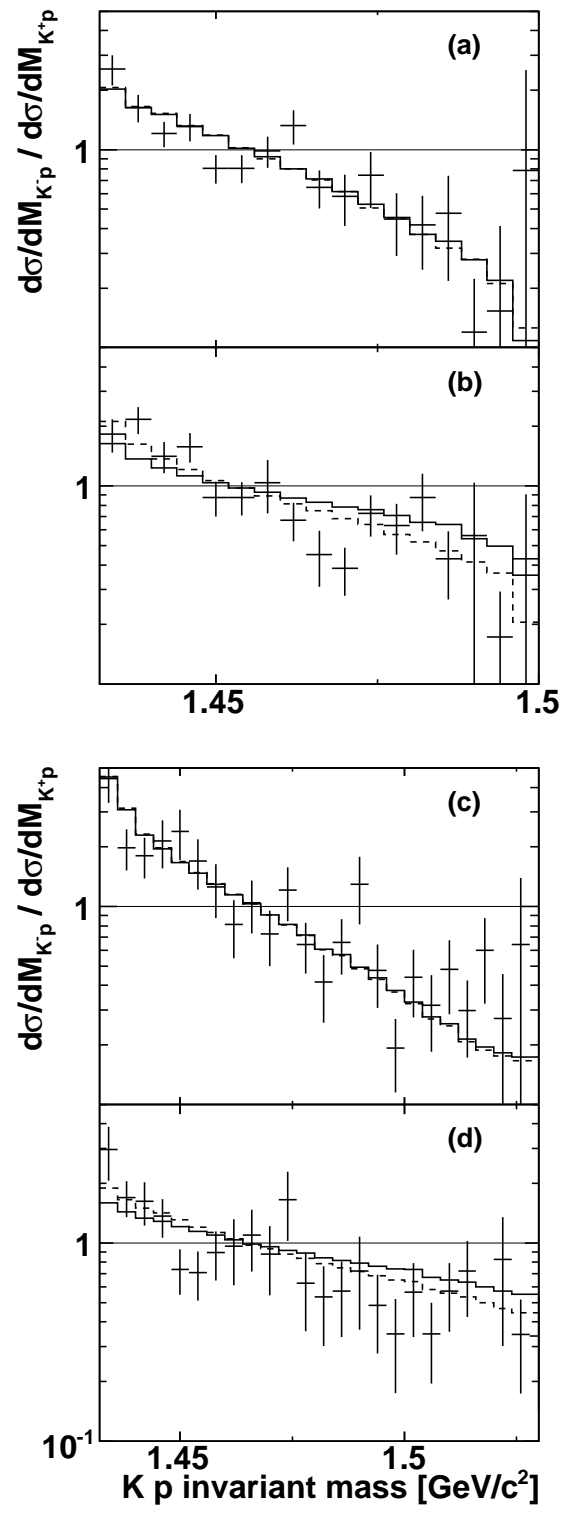

FIG. 8: Values of the $R_{K p}$ ratio for (a) the $\phi$-poor region and (b) the $\phi$-rich at $2.70 \mathrm{GeV}$ with (c) and (d) showing respectively the analogous results at $2.83 \mathrm{GeV}$. The predictions obtained using the standard parameters are shown with no fsi effect for the $K^{-}$from the decay of the $\phi$ (solid curve) and $20 \%$ (dashed).

shown by the $28 \mathrm{MeV}$ data is convincing. Unfortunately, in the publication [9] the values of the ratio at $10 \mathrm{MeV}$ were scaled upwards to make them lie closer to those taken at $28 \mathrm{MeV}$. Since the numbers of $K^{-} p$ and $K^{+} p$ events are the same, the weighted average of the ratio must be unity and this allows one to make an informed guess regarding the value of the scaling that has to be reintroduced. No account has been taken of the COSY11 invariant-mass resolution, which will be of greater significance for the $10 \mathrm{MeV}$ data. 

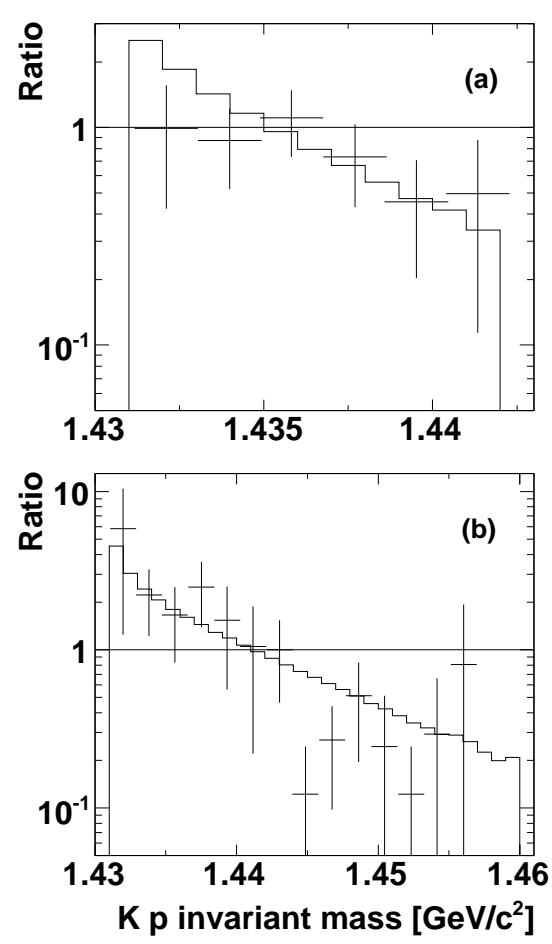

FIG. 9: The $R_{K p}$ ratio for the COSY-11 $p p \rightarrow p p K^{+} K^{-}$data at (a) $\varepsilon=10 \mathrm{MeV}$ (upper panel) and (b) $\varepsilon=28 \mathrm{MeV}$. The small contribution from the low mass tail of the $\phi$ has been neglected in the $28 \mathrm{MeV}$ simulation. Since the $10 \mathrm{MeV}$ results were arbitrarily scaled in the publication [9], they have here been divided by a factor of three before being compared to the predictions of the $f$ si model, using the same values of the parameters as for the ANKE data.

\section{THE KAON-PROTON-PROTON INVARIANT-MASS DISTRIBUTION}

We have argued in Sect. IV and Appendix A that it is natural to take the final state enhancement to be the product of the individual factors corresponding to the $p p$ and two $K^{-} p$ interactions, which allows the kaon to be attracted simultaneously to both protons. This in turn suggests that there might also be an enhancement in the three-body system at low $K^{-} p p$ invariant masses. This is indeed the case. The size of the effect at $2.65 \mathrm{GeV}$ is illustrated in Fig. 10 for the regions with $M\left(K^{+} K^{-}\right) \gtrless$ $1.01 \mathrm{GeV} / \mathrm{c}^{2}$.

There is a striking preference for low Kpp masses in both $K^{+} K^{-}$intervals and this behavior is reproduced quantitatively by our simulation, which uses the same $K^{-} p$ effective scattering length $a$ that was chosen to describe the two-body $R_{K p}$ ratio. It provides some empirical justification for our choice of the final-stateinteraction enhancement factor as the product of the individual two-body enhancements. This favors configurations where the $K^{-}$is attracted simultaneously to both protons. It must, however, be stressed that the strong mass dependence which is apparent in Fig. 10 does not necessarily imply that the $K^{-}$forms a bound state with the two protons $20,21,22]$.

Though the error bars are considerably larger at the two higher energies, as seen in Fig. 11, the simulation is consistent with the results. In all these three-particle mass distributions the introduction of a small amount of $f_{s i}$ for the products of the $\phi$ decay seems to be of less importance than for the $K p$ spectra.

\section{TOTAL CROSS SECTIONS}

The total cross section results for both $\phi$ and non$\phi$ production are given in Table $\llbracket$ for the three energies. The systematic errors given here arise from the quadratic sum of the uncertainties in the background subtraction, acceptance correction, tracking efficiency correction for kaons $(3 \%)$ and the analysis of the $p p$-elastic data used for the normalization. Unfortunately, the $p p$ database is very limited in our region of angle and energy. This is recognized in the recent SP07 update to the SAID program where it is stated that our solution should be considered at best qualitative between 2.5 and $3 \mathrm{GeV}$ [23]. The raw SP07 solution generally overestimates the values of those $p p$ differential cross sections that are available in this range 24]. A normalization option has to be chosen to ensure agreement with the $p p$ experimental data and this typically leads to reductions of about $15 \%$ at our two lower energies but almost $20 \%$ at $2.83 \mathrm{GeV}$. To eliminate any ambiguities, the values that we have assumed for the $p p$ elastic cross section, integrated over the solid angle defined by $5.0^{\circ}<\theta_{\text {lab }}<8.5^{\circ}$, are presented in Table 【 for our three energies.

TABLE I: Total cross sections for the $p p \rightarrow p p\left\{K^{+} K^{-}\right\}_{\text {non- } \phi}$ and $p p \rightarrow p p \phi$ reactions at three beam energies $T_{p}$ and corresponding excess energies $\varepsilon_{K K}$ with respect to the $p p K^{+} K^{-}$ threshold. The excess energy with respect to the $\phi$ threshold is given by $\varepsilon_{\phi}=\varepsilon_{K K}-32.1 \mathrm{MeV}$. The $\phi$ cross section has been corrected for the $49.1 \%$ branching ratio [4]. For both channels the first error is statistical and the second systematic. The values that we have taken for $\sigma_{p p}$, the integral of the proton-proton elastic cross section over the solid angle range $5.0^{\circ}<\theta_{\text {lab }}<8.5^{\circ}$, are also given. The associated $\approx \pm 6 \%$ uncertainty has not, however, been compounded with the other errors.

\begin{tabular}{c|c|c|c|c}
\hline \hline$T_{p}$ & $\varepsilon_{K^{+} K^{-}}$ & $\sigma_{p p}$ & $\sigma_{\text {non- } \phi}($ tot $)$ & $\sigma_{\phi}($ tot $)$ \\
{$[\mathrm{GeV}]$} & {$[\mathrm{MeV}]$} & {$[\mathrm{mb}]$} & {$[\mathrm{nb}]$} & {$[\mathrm{nb}]$} \\
\hline 2.65 & 51 & 5.52 & $16 \pm 1 \pm 1$ & $33 \pm 2 \pm 4$ \\
2.70 & 67 & 5.44 & $30 \pm 2 \pm 3$ & $64 \pm 4 \pm 10$ \\
2.83 & 108 & 5.06 & $98 \pm 8 \pm 15$ & $133 \pm 12 \pm 27$ \\
\hline \hline
\end{tabular}

The value that we obtain for the luminosity at $2.65 \mathrm{GeV}$ on the basis of the $p p$ cross section given in the table could be checked using a novel technique [25]. This depends upon the energy loss of the coasting proton beam caused by its multiple traversals through the 

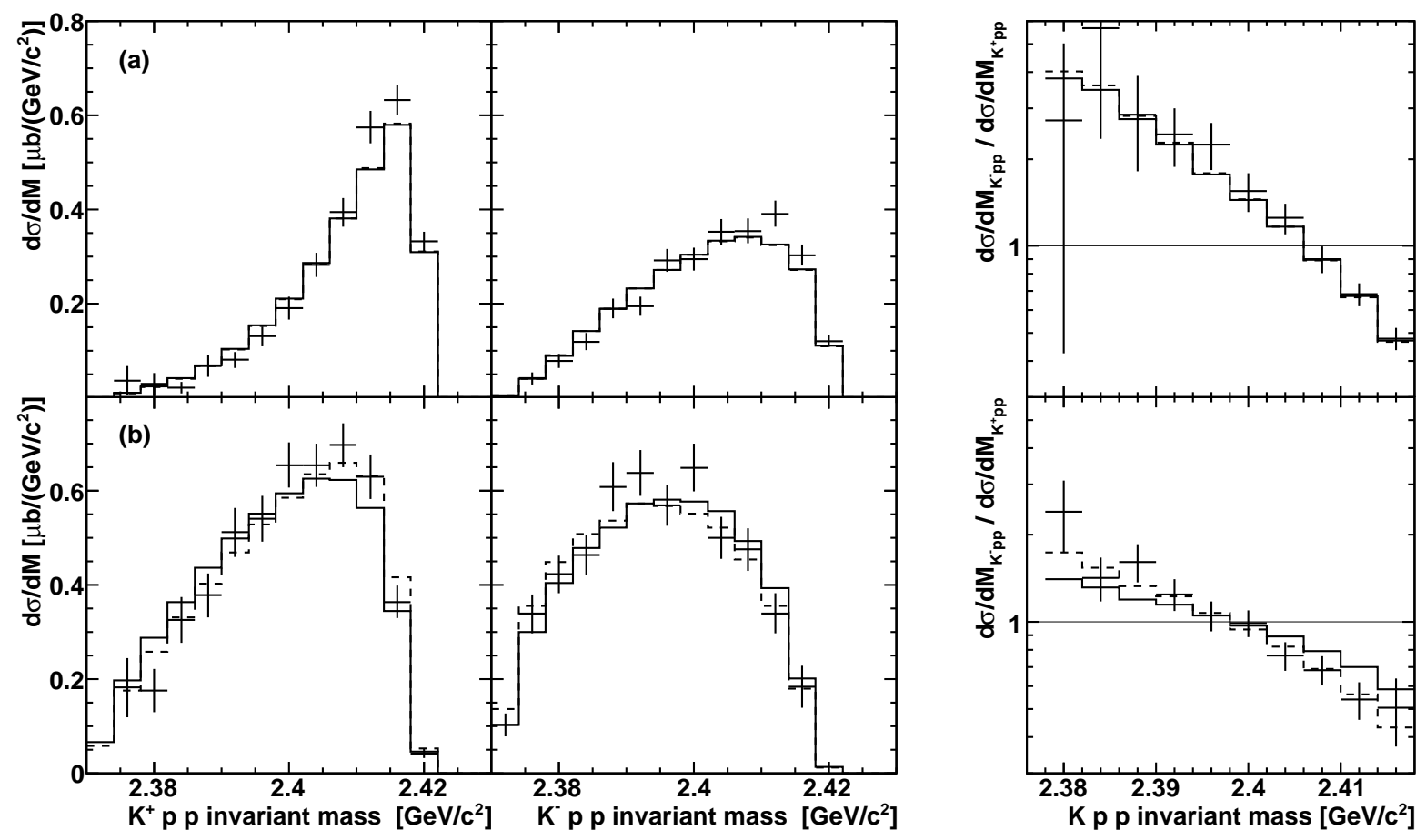

FIG. 10: Differential cross sections for the $p p \rightarrow p p K^{+} K^{-}$reaction at $2.65 \mathrm{GeV}$ with respect to the invariant masses of the $K^{+} p p$ and the $K^{-} p p$ systems and their ratio. The data are divided (a) into a $\phi$-poor region with $M\left(K^{+} K^{-}\right)<1.01 \mathrm{GeV} / \mathrm{c}^{2}$ (upper panels) and (b) $\phi$-rich with $M\left(K^{+} K^{-}\right)>1.01 \mathrm{GeV} / \mathrm{c}^{2}$ (lower panels). The predictions obtained using the standard parameters are shown with no $f s i$ effect for the $K^{-}$from the decay of the $\phi$ (solid curve) and $20 \%$ (dashed). The simulations that describe the two-particle ratios also represent well these three-body ratios.

very thin target. The resultant frequency change can be measured with high accuracy using the spectrum of the Schottky noise. Combined with measurements of the beam current, this yielded luminosities with an expected precision of about $\pm 6 \%$. The two methods give consistent results within this uncertainty and, although the frequency-change technique could not be applied as reliably at the higher energies, it is expected that the overall luminosity uncertainty is on about this level.

Our total cross section results for non- $\phi$ production are plotted in Fig. 12 along with results taken from DISTO [1] and COSY-11 [9, 26, 27]. It is seen that four-body phase space cannot describe simultaneously the $p p \rightarrow p p K^{+} K^{-}$data at high and low excess energies. The situation is improved only a little if account is taken of the strong attraction between the two protons in the ${ }^{1} S_{0}$ state but a greater improvement is achieved through the introduction of the $f s i$ in the $K^{-} p$ system. Nevertheless, the COSY-11 data at excess energies of $20 \mathrm{MeV}$ or less still seem to be underestimated. This might be connected to the incomplete description of the $K^{+} K^{-}$ spectra of Fig. 3 at very low invariant masses. However, it must be stressed that in the evaluation of the COSY-11 acceptance no account was taken of any $K^{-} p f s i$.

\section{CONCLUSIONS AND OUTLOOK}

We have investigated the $p p \rightarrow p p K^{+} K^{-}$reaction at three energies. In those parts of the $K^{+} K^{-}$mass spectra where $\phi$ production is small, we find a marked difference between the $K^{-} p$ and $K^{+} p$ invariant-mass distributions. There is a strong peaking in the $K^{-} p / K^{+} p$ ratio towards the $K p$ threshold for both the COSY-11 and our data. This clearly indicates that the $p p \rightarrow p p K^{+} K^{-}$reaction cannot be dominated by the undistorted production of a single scalar resonance $a_{0}$ or $f_{0}$. Either a mixture of the two is produced or one of the kaons from the $a_{0} / f_{0}$ decay interacts with a final proton. As we have shown, these data can be explained quantitatively through the introduction of a simple $K^{-} p$ final state interaction.

In kinematic regions where $\phi$ production is significant, the $K^{-} p / K^{+} p$ asymmetry is reduced because the meson may travel some distance before decaying. Our data in such conditions are well fit by assuming that about $20 \%$ of the $K^{-}$from the $\phi$ decay interact with one or both final protons. The uncertainty in this is, however, quite large because of the limited precision with which the $\phi$ contribution to the $K^{+} K^{-}$mass spectrum can be isolated.

There is no universal technique for modelling the $f s i$ when more than one pair of particles is involved. The 

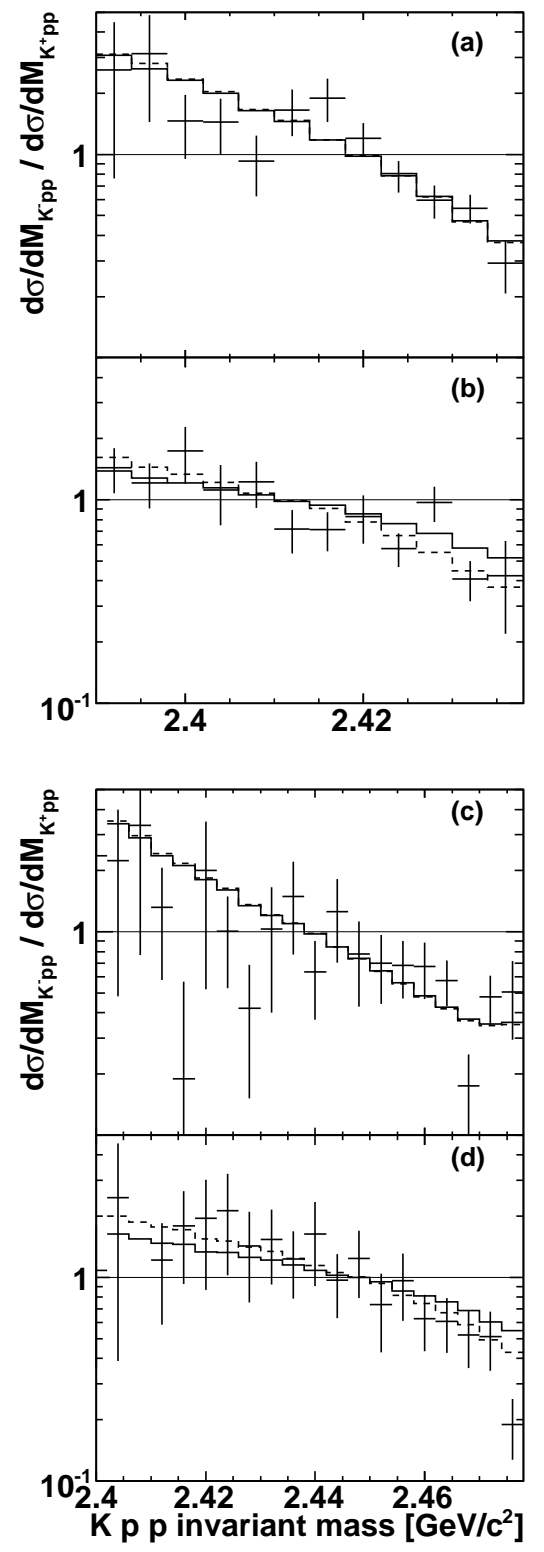

FIG. 11: The ratio of the differential cross sections of the $p p \rightarrow p p K^{+} K^{-}$reaction with respect to the $K^{-} p p$ to $K^{+} p p$ invariant masses for (a) the $\phi$-poor region and (b) the $\phi$-rich at $2.70 \mathrm{GeV}$ with (c) and (d) showing respectively the analogous results at $2.83 \mathrm{GeV}$. The predictions obtained using the standard parameters are shown with no $f$ si effect for the $K^{-}$ from the decay of the $\phi$ (solid curve) and 20\% (dashed). The simulations that describe the two-particle ratios also represent well these three-body ratios.

product ansatz has the benefit that the same simulation with the same parameters reproduces also the strong peaking in the ratio of the $K^{-} p p / K^{+} p p$ mass distributions.

We find an effective $K^{-} p$ scattering length that is of the same order of magnitude as that needed to describe the free scattering data [19]. However, the error bars are

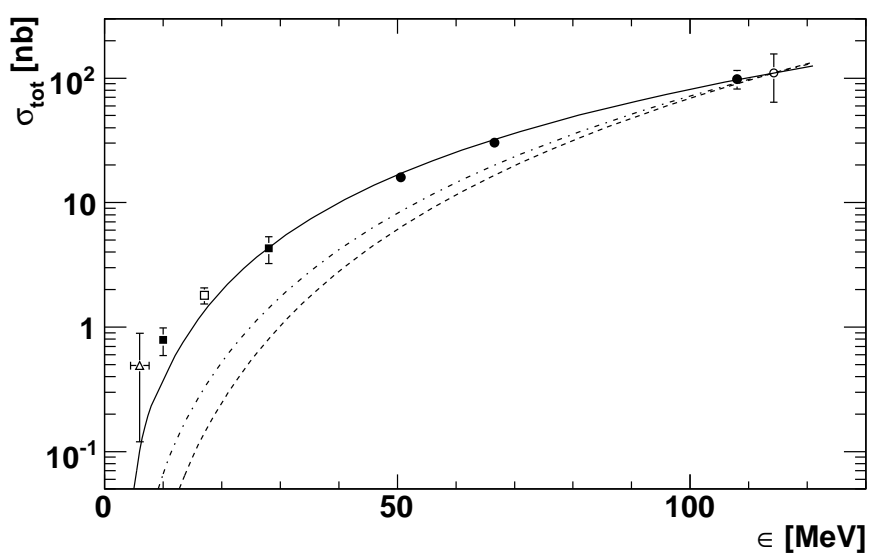

FIG. 12: Energy dependence of the non- $\phi$ contribution to the $p p \rightarrow p p K^{+} K^{-}$total cross section. In addition to our three points (closed circles), there is a high energy point from DISTO (open circle) 1] and four points close to threshold from COSY-11 (closed squares) [9], (open triangle) [26], and (open square) [27]. The dashed curve represents the energy dependence from four-body phase space. The dot-dashed includes the effects of the $p p$ final state interaction whereas the solid curve contains in addition distortion from the $K^{-} p f s i$, as described in the text.

large and our data are primarily sensitive to $|a|$ rather than its phase. It is important to stress that we do not know the relative strengths for the production of isospin $I=0$ and $I=1 K^{-} p$ pairs in the $p p \rightarrow p p K^{+} K^{-}$reaction and hence the relative weights of $a_{0}$ and $a_{1}$. Some information on this might come from looking at the data on the $p p \rightarrow K^{+} p \Sigma^{0}(1385)$ and $p p \rightarrow K^{+} p \Lambda(1405)$ reactions taken at $2.83 \mathrm{GeV}[8]$. However, even this would not give access to the phase between the $I=0$ and $I=1$ amplitudes. It must also be stressed that we have neglected any energy dependence of the parameter $a$. Another important caveat is that we have not considered any possible interaction of the $K^{+}$with the protons. Although there is no evidence for any strong effect of this nature in the $p p \rightarrow K^{+} \Lambda p$ reaction [28], since the $K^{+} p$ interaction is repulsive, its neglect might be interpreted as extra attraction in the $K^{-} p$ system.

We have clearly shown the importance of the $K^{-} p$ interaction, which is possibly related to the $\Lambda(1405)$ or the $\Sigma^{0}(1385)$, but we must now ask whether there is any evidence for effects that might be connected with the production of the $a_{0} / f_{0}$ resonances. The simulation of the energy dependence of the total cross section for the production of the non- $\phi$ component in the $p p \rightarrow p p K^{+} K^{-}$reaction underestimates the COSY-11 data very near threshold. Even more intriguing, and possibly connected, are the lowest mass points in the $K^{+} K^{-}$spectra. At all three energies shown in Fig. 3, these points lie much higher than the simulations and a similar behavior is visible in the DISTO results [1] as well as in our data on the $p n \rightarrow d K^{+} K^{-}$reaction [3]. The 
mass scale of the variation is not that of the widths of the scalar resonances, which are quite large. It is tempting to suggest that this structure might be due to the opening of the $K^{0} \bar{K}^{0}$ channel at a mass of $\approx 8 \mathrm{MeV} / \mathrm{c}^{2}$, which induces some cusp structure that changes the energy dependence of the total cross section near threshold [9, 29]. This would require a very strong $K^{+} K^{-} \rightleftharpoons K^{0} \bar{K}^{0}$ channel coupling, which might be driven by the $a_{0} / f_{0}$ resonances. Thus, although the $p p \rightarrow p p K^{+} K^{-}$reaction may not be ideal for investigating the properties of scalar states, their indirect effects might still be crucial.

Given that the $K^{-}$is strongly attracted to protons, it is natural to expect that similar $f_{s i}$ effects should exist for other reactions. The $\bar{K}^{0} d / K^{+} d$ ratio in the $p p \rightarrow K^{+} \bar{K}^{0} d$ reaction does indeed show a very strong enhancement near the $K d$ threshold 7]. The interpretation is, however, slightly more complex because spinparity constraints means that there must be at least one $p$-wave in the final state, even at low energies. Unfortunately, the MOMO data on $p d \rightarrow K^{+} K^{-3} \mathrm{He}$ [30] cannot be used to isolate any $K^{-3} \mathrm{He}$ interaction because the charges of the kaons were not identified and only averaged spectra could be studied [31].

There is currently an overall uncertainty in our values of the cross sections because of the limitation in the proton-proton elastic scattering database in our region of energy and angle, though we hope that this will be alleviated by future measurements [25]. With our new estimates for the luminosity, the $\phi$-production cross sections are reduced compared to those of our first analysis [2], which relied upon the non-renormalized 2004 SAID solution [23]. Furthermore, the better separation of the $\phi$ and non- $\phi$ events goes by chance in the same direction. These two effects lower the ratio of $\phi$ to $\omega$ production in proton-proton collisions near threshold so that it is now only about a factor of six above the Okubo-Zweig-Iizuka limit 32].

\section{Acknowledgments}

We would like to thank the COSY machine crew for their support as well as that of other members of the ANKE Collaboration. Discussions with J. Haidenbauer, C. Hanhart and A. Sibirtsev were very helpful and those with I. Strakovsksy provided important clarifications on the uncertainties in the SAID $p p$ procedure above $2.5 \mathrm{GeV}$. R. Nißler and U.-G. Meißner kindly provided us with the predictions of Ref. [19] in numerical form. Correspondence with R. Bertini and P. Winter is gratefully acknowledged. This work has been supported in part by the BMBF, DFG, Russian Academy of Sciences, and COSY FFE.

\section{APPENDIX A: FINAL STATE INTERACTIONS}

When two particles interact strongly in the final state, the resulting matrix element involves an average of a production operator with the relative wave function $\psi_{\vec{q}}(\vec{r})$ of the strongly interacting pair. If the interaction is of very short range, the wave function may be evaluated at the origin to leave an enhancement factor

$$
F_{2}(q) \propto \psi_{\vec{q}}(0) \propto \frac{1}{D(q)},
$$

where $\vec{q}$ is the relative momentum in the pair and $D(q)$ is the $S$-wave Jost function [18]. In the commonly used scattering length approximation, one retains only the linear term in $q$, in which case $F(q)=1 /(1-i q a)$, where $a$ is the scattering length of the interacting pair.

On the other hand, if two or more pairs of particles interact in the final state, there is no reliable prescription to evaluate an analogous enhancement factor since a three-body equation then needs to be solved. If we denote the interacting pairs as 12 and 13, the corresponding wave function will be $\Psi_{\vec{q}_{12}, \vec{q}_{13}}\left(\vec{r}_{12}, \vec{r}_{13}\right)$. We now make the ad hoc assumption that this wave function factorizes in the form

$$
\Psi_{\vec{q}_{12}, \vec{q}_{13}}\left(\vec{r}_{12}, \vec{r}_{13}\right)=\psi_{\vec{q}_{12}}\left(\vec{r}_{12}\right) \times \psi_{\vec{q}_{13}}\left(\vec{r}_{13}\right) .
$$

In this case, the three-body enhancement factor is simply the product of the two-body factors, evaluated at the appropriate relative momenta:

$$
\begin{aligned}
F_{3}\left(q_{12}, q_{13}\right) & =F_{2}\left(q_{12}\right) \times F_{2}\left(q_{13}\right) \\
& \approx \frac{1}{\left(1-i q_{12} a_{12}\right)\left(1-i q_{13} a_{13}\right)} .
\end{aligned}
$$

It must be stressed that Eq. (A33 is merely an ansatz to try to understand our ensemble of data and, even if it provides a satisfactory description of these, this does not mean that it is applicable more generally. However, it has also been used to treat the $p p \rightarrow p p \eta$ reaction near threshold, where all the final pairs of particles interact strongly [17].

The approach does retain the necessary pole structure when $q_{12}=-i / a_{12}$ and similarly for $q_{13}$. Thus particle1 can interact simultaneously with both 2 and 3. Furthermore, to lowest order in the momenta, the ansatz corresponds to the scattering length of particle- 1 from a composite $2+3$ with the desired combined scattering length of $a=a_{12}+a_{13}$.

Although the $S$-wave $p p$ interaction is well known, the $K^{-} p$ interaction is far more complex because of the channel couplings to $\Sigma \pi$ and also because there are two isospins $I=0$ and $I=1$. In a recent study, within a chiral $\mathrm{SU}(3)$ unitary approach [19], $K^{-} p$ data leading to a variety of channels have been fit and values of the (complex) $K^{-} p$ scattering lengths deduced for $I=0$ and $I=1$. Their full model resulted in

$$
\begin{aligned}
& a_{0}=(-1.64+i 0.75) \mathrm{fm}, \\
& a_{1}=(-0.06+i 0.57) \mathrm{fm} .
\end{aligned}
$$


These values describe the rapid drop in the $K^{-} p$ elastic amplitudes from threshold.

If isospin breaking induced by the significant mass differences is neglected, the $K^{-} p$ scattering length $a_{K^{-}}{ }=$ $\left(a_{0}+a_{1}\right) / 2$. However, this is not necessarily the parameter that is relevant for the analysis of the $p p \rightarrow$ $p K^{+}\left\{K^{-} p\right\}$ because it is not clear whether the reaction mechanism preferentially excites $I=0$ or $I=1$ $\left\{K^{-} p\right\}$ states or some combination thereof. The values in Eq. (A4) should therefore be considered merely as orderof-magnitude estimates of what might be expected when making fits to our data.
[1] F. Balestra et al., Phys. Rev. C 63, 024004 (2001).

[2] M. Hartmann et al., Phys. Rev. Lett. 96, 242301 (2006).

[3] Y. Maeda et al., Phys. Rev. Lett. 97, 142301 (2006).

[4] W.-M. Yao et al., J. Phys. G 33, 1 (2006).

[5] See the short note on scalar mesons by S. Spannier and N.A Törnquist in S. Eidelman et al., Phys. Lett. B 592, 506 (2004).

[6] J. Weinstein and N. Isgur, Phys. Rev. D 41, 2236 (1990).

[7] V. Kleber et al., Phys. Rev. Lett. 91, 172304 (2003); A. Dzyuba et al., Eur. Phys. J. A 29, 245 (2006).

[8] I. Zychor et al., arXiv:0705.1039[nucl-ex].

[9] P. Winter et al., Phys. Lett. B 635, 23 (2006).

[10] S. Barsov et al., Nucl. Instrum. Meth. Phys. Res., Sect. A 462, 364 (2001).

[11] R. Maier et al., Nucl. Instrum. Meth. Phys. Res., Sect. A 390, 1 (1997).

[12] A. Khoukaz et al., Eur. Phys. J. D 5, 275 (1999).

[13] S. Dymov et al., Part. Nucl. Lett. 2, 40 (2004).

[14] M. Hartmann et al., Int. J. Mod. Phys. A 22, 317 (2007).

[15] M. Büscher et al., Nucl. Instrum. Meth. Phys. Res., Sect. A 481, 378 (2002).

[16] S. Agostinelli et al., Nucl. Instrum. Meth. A 506, 250 (2003); http://geant4.web.cern.ch/geant4.

[17] V. Bernard, N. Kaiser, and U.-G. Meißner, Eur. Phys. J. A 4, 259 (1999).

[18] M.L. Goldberger and K.M. Watson, Collision Theory, (Wiley, N.Y., 1964) p. 544.

[19] B. Borasoy, U.-G. Meißner, and R. Nißler, Phys. Rev. C 74, 055201 (2006).
[20] M. Agnello et al., Phys. Rev. Lett. 94, 212303 (2005).

[21] T. Yamazaki and Y. Akaishi, arXiv:0709.0630[nucl-th]

[22] N.V. Shevchenko, A. Gal, J. Mareš, J. Révai, arXiv:0706.4393[nucl-th]

[23] R.A. Arndt, I.I. Strakovsky, and R.L. Workman, Phys. Rev. C 62, 034005 (2000); R.A. Arndt, W.J. Briscoe, I.I. Strakovsky, and R.L. Workman, Phys. Rev. C 76, 025209 (2007); SAID solution SP07, available from http://gwdac.phys.gwu.edu

[24] D. Albers et al., Eur. Phys. J. A 2, 125 (2004); R.C. Kammerud et al., Phys. Rev. D 4, 1309 (1971); S.A. Azimov et al., Sov. Phys. - JETP 15, 299 (1962); I. Ambats et al., Phys. Rev. D 9, 1179 (1974); T. Fujii et al., Phys. Rev. 128, 1836 (1962); W.M. Preston, R. Wilson, and J.C. Street, Phys. Rev. 118, 579 (1960).

[25] H.J. Stein et al., in preparation.

[26] M. Wolke, PhD thesis, University of Münster (1997).

[27] C. Quentmeier et al., Phys. Lett. B 515, 276 (2001).

[28] S. Abd El-Samad et al., Phys. Lett. B 632, 27 (2006).

[29] W. Oelert et al., Int. J. Mod. Phys. A 22, 502 (2007).

[30] F. Bellemann et al., Phys. Rev. C 75, 015204 (2007).

[31] V.Yu. Grishina, M. Büscher, and L.A. Kondratyuk, Phys. Rev. C 75, 015208 (2007).

[32] S. Okubo, Phys. Lett. 5, 165 (1963); G. Zweig, CERN report TH-401 (1964); J. Iizuka, Prog. Theor. Phys. Suppl. 38, 21 (1966). 